\title{
Frequentist nonparametric goodness-of-fit tests via marginal likelihood ratios
}

\author{
Jeffrey D. Hart ${ }^{\mathrm{a}}$, Taeryon Choi ${ }^{\mathrm{b}, *}$, Seongbaek Yi ${ }^{\mathrm{c}}$ \\ ${ }^{a}$ Department of Statistics, Texas A\&M University, U.S.A. \\ ${ }^{b}$ Department of Statistics, Korea University, Republic of Korea \\ ${ }^{c}$ Department of Statistics, Pukyoung National University, Republic of Korea
}

\begin{abstract}
A nonparametric procedure for testing the goodness of fit of a parametric density is investigated. The test statistic is the ratio of two marginal likelihoods corresponding to a kernel estimate and the parametric model. The marginal likelihood for the kernel estimate is obtained by proposing a prior for the estimate's bandwidth, and then integrating the product of this prior and a leave-one-out kernel likelihood. Properties of the kernel-based marginal likelihood depend importantly on the kernel used. In particular, a specific, somewhat heavy-tailed, kernel $K_{0}$ yields better performing marginal likelihood ratios than does the popular Gaussian kernel. Monte Carlo is used to compare the power of the new test with that of the Shapiro-Wilk test, the Kolomogorov-Smirnov test, and a recently proposed goodness-of-fit test based on empirical likelihood ratios. Properties of these tests are considered when testing the fit of normal and double exponential distributions. The new test is used to establish a claim made in the astronomy literature concerning the distribution of nebulae brightnesses in the Andromeda galaxy. Generalizations to the multivariate case are also described.
\end{abstract}

Keywords: Bandwidth parameter, Empirical null distribution, Goodness-of-fit tests, Kernel density estimation, Marginal likelihoods

\section{Introduction}

We consider the classical problem of testing the goodness-of-fit of a parametric model for a distribution. Our approach is nonparametric in that our

\footnotetext{
*Corresponding Author

Email : trchoi@gmail.com, Tel : +82-2-3290-2245, Fax : +82-2-924-9895
} 
test can consistently detect virtually any departure from the null hypothesis. The proposed test statistic is based on the ratio of two marginal likelihoods, in which the alternative "model" corresponds to a kernel density estimator. There exist a number of well-known goodness-of-fit tests including Neyman's smooth test (Neyman, 1937), the Kolomogorov-Smirnov test (Smirnov, 1944), the Anderson-Darling test (Anderson and Darling, 1954) and, for testing normality, the Shapiro-Wilk test (Shapiro and Wilk, 1965). Additionally, several recent developments have been made in the literature of nonparametric goodness-of-fit testing, see, e.g., Claeskens and Hjort (2004) for a goodness-of-fit test based on nonparametric likelihood ratios, and, more recently, Vexler and Gurevich (2010) for a goodness-of-fit test based on sample entropy and empirical likelihood ratios. The reader is also referred to the monographs of Hart (1997), Ingster and Suslina (2003), Rayner et al. (2009) and Thas (2010) and references therein for general theory and methods in the treatment of goodness-of-fit and lack-of-fit problems.

Suppose that we have independent and identically distributed observations $\boldsymbol{X}_{n}=\left(X_{1}, \ldots, X_{n}\right)$, and wish to compare the fit of two candidate models $\mathcal{M}_{0}$ and $\mathcal{M}_{1}$ for the distribution of $X_{i}$ :

$$
\mathcal{M}_{0}=\left\{f_{0}(\cdot \mid \boldsymbol{\theta}): \boldsymbol{\theta} \in \Theta\right\} \text { and } \mathcal{M}_{1}=\left\{f_{1}(\cdot \mid \boldsymbol{\lambda}): \boldsymbol{\lambda} \in \Lambda\right\},
$$

where $f_{0}$ and $f_{1}$ are known up to their respective parameter vectors.

The marginal likelihood $m\left(\boldsymbol{X}_{n}\right)$ is obtained by integrating the product of the likelihood and a prior over the parameter space. Given prior distributions $\pi_{0}$ and $\pi_{1}$ for $\boldsymbol{\theta}$ and $\boldsymbol{\lambda}$, respectively, the marginal likelihoods $m_{0}\left(\boldsymbol{X}_{n}\right)$ and $m_{1}\left(\boldsymbol{X}_{n}\right)$ are given by

$$
m_{0}\left(\boldsymbol{X}_{n}\right)=\int \prod_{i=1}^{n} f_{0}\left(X_{i} \mid \boldsymbol{\theta}\right) \pi_{0}(\boldsymbol{\theta}) d \boldsymbol{\theta}
$$

and

$$
m_{1}\left(\boldsymbol{X}_{n}\right)=\int \prod_{i=1}^{n} f_{1}\left(X_{i} \mid \boldsymbol{\lambda}\right) \pi_{1}(\boldsymbol{\lambda}) d \boldsymbol{\lambda} .
$$

The ratio $m_{0}\left(\boldsymbol{X}_{n}\right) / m_{1}\left(\boldsymbol{X}_{n}\right)$ is known as a Bayes factor (see, e.g., Jeffreys (1961) and Kass and Raftery (1995)), and represents the ratio of the prior and posterior odds of the two models. On the other hand, it may also be used in frequentist fashion to test the fit of one model versus another, an idea which appears to be due to Good (1957), who referred to the idea as a Bayes/non-Bayes synthesis(compromise). Good (1967) proposed using the null distribution of a Bayes factor as a significance criterion. An extensive 
review of such approaches is given by Good (1992). Recently Aerts et al. (2004) and Hart (2009) proposed Bayesian-motivated frequentist tests for lack of fit in the context of regression.

Our paper likewise proposes a Bayes/non-Bayes synthesis for nonparametric goodness-of-fit testing in which we use as test statistic a marginal likelihood ratio comparing a parametric model to essentially arbitrary alternatives. The alternative is estimated by a kernel density estimate, whose only parameter is its bandwidth. We therefore propose a prior distribution for the bandwidth and obtain a nonparametric marginal likelihood by integrating the product of this prior and a leave-one-out type of kernel likelihood. A traditional marginal likelihood is computed for the parametric null model. It is shown that the proposed nonparametric goodness-of-fit test using the marginal likelihood ratio is easily computed and powerful in detecting departures from the null hypothesis.

The remainder of the paper proceeds as follows. Our basic approach is described in Section 2. This includes how we choose kernels and prior distributions for the bandwidth, and a statement of conditions guaranteeing that the nonparametric marginal likelihood exists finite. Monte Carlo studies of power when testing normality and double exponentiality, and an application of our approach to astronomy data are presented in Section 3. Extensions to multivariate distributions are described in Section 4, and concluding remarks given in Section 5 .

\section{Basic Approach: Marginal Likelihood Ratio}

Let $\boldsymbol{X}_{n}=\left(X_{1}, \ldots, X_{n}\right)$ be a random sample of size $n$ from an unknown probability density $f$. We wish to test the following hypotheses:

$$
H_{0}: f \in \mathcal{F}_{0}=\left\{f_{0}(\cdot \mid \boldsymbol{\theta}) \mid \boldsymbol{\theta} \in \Theta\right\} \quad \text { vs. } \quad H_{1}: f \notin \mathcal{F}_{0},
$$

where $f_{0}(\cdot \mid \boldsymbol{\theta})$ is known up to the vector of parameters $\boldsymbol{\theta}=\left(\theta_{1}, \ldots, \theta_{p}\right)$, which is unknown. Note that $\mathcal{F}_{0}$ is a completely specified parametric family of distributions, e.g., the normal distribution with mean $\theta_{1}$ and variance $\theta_{2}$, $\boldsymbol{\theta}=\left(\theta_{1}, \theta_{2}\right)$. The alternative is nonparametric, in that it contains densities not in $\mathcal{F}_{0}$.

As indicated previously, our test statistic is a marginal likelihood ratio that consists of a parametric marginal likelihood for the null model and a nonparametric marginal likelihood based on kernel density estimates for the alternative model. In particular, we consider the following cross-validated 
likelihood under $H_{1}$ :

$$
L\left(h \mid \boldsymbol{X}_{n}\right)=\prod_{i=1}^{n} \widehat{f}_{i}\left(X_{i} \mid h\right), \quad \widehat{f}_{i}(x \mid h)=\frac{1}{(n-1) h} \sum_{j \neq i} K\left(\frac{x-X_{j}}{h}\right),
$$

where $h>0$ is a bandwidth and $K$ a suitable kernel function, a popular choice of which is the Gaussian kernel, i.e., $K \equiv \phi$, with $\phi(z)=$ $(2 \pi)^{-1 / 2} \exp \left(-\frac{1}{2} z^{2}\right)$. The kernel estimates $\widehat{f}_{i}(x \mid h), i=1, \ldots, n$, (Marron (1985)) are known as cross-validated or leave-one-out estimates, inasmuch as $\widehat{f}_{i}(x \mid h)$ is computed without $X_{i}$. It is well-known that $\widehat{f}_{i}(x \mid h)$ is consistent for $f(x)$ (Chow et al., 1983) under mild conditions, including $n h \rightarrow \infty$ and $h \rightarrow 0$ as $n \rightarrow \infty$. Typically $L\left(h \mid \boldsymbol{X}_{n}\right)$ is used as a bandwidth selection criterion, but we use it to construct a nonparametric marginal likelihood under the alternative hypothesis $H_{1}$.

Let $\pi_{\mathrm{bw}}$ be a prior for the bandwidth $h$ and $\pi_{0}$ a prior for $\boldsymbol{\theta}$, both of which are assumed to be proper. Define the following marginal likelihood ratio $\left(\mathrm{MLR}_{n}\right)$ based on $n$ observations:

$$
\operatorname{MLR}_{n} \equiv \operatorname{MLR}\left(\boldsymbol{X}_{n}\right)=\frac{\int_{0}^{\infty} L\left(h \mid \boldsymbol{X}_{n}\right) \pi_{\mathrm{bw}}(h) d h}{\int_{\Theta} \Pi_{i=1}^{n} f_{0}\left(X_{i} \mid \boldsymbol{\theta}\right) \pi_{0}(\boldsymbol{\theta}) d \boldsymbol{\theta}}
$$

As noted in the previous section, $\mathrm{MLR}_{n}$ may be used in frequentist fashion to test $H_{0}$. Thus, one must approximate the null distribution of $\mathrm{MLR}_{n}$ (which is not analytically available in general), and then reject $H_{0}$ at level $\alpha$ if the observed value of $\mathrm{MLR}_{n}$ exceeds the $(1-\alpha)$ percentile of the null distribution.

\subsection{Choice of prior distribution}

A desirable property of any goodness-of-fit statistic is that its distribution be invariant to unknown parameters under the null hypothesis. While $\mathrm{MLR}_{n}$ cannot be expected to have this property in general, it is of interest to investigate whether the property will at least hold when testing the fit of a location-scale family of densities. Suppose that

$$
\mathcal{M}_{0}=\left\{\frac{1}{\sigma} f_{0}\left(\frac{x-\mu}{\sigma}\right):-\infty<\mu<\infty, \sigma>0\right\},
$$

where $f_{0}$ is a known, completely specified density. Then if the density of $X_{i}$ is in $\mathcal{M}_{0}$, we desire that the distribution of $\mathrm{MLR}_{n}$ be the same regardless of the true value, $\left(\mu_{0}, \sigma_{0}\right)$, of $(\mu, \sigma)$. 
One way to make $\mathrm{MLR}_{n}$ location-scale invariant when $\mathcal{M}_{0}$ is a locationscale family is by using proper priors that are tuned appropriately to the data. We use data-tuned priors that contain a very small amount of information relative to the data. Define $\pi_{\mathrm{bw}}(\cdot \mid \beta)$ by

$$
\pi_{\mathrm{bw}}(h \mid \beta)=\frac{\sqrt{\beta}}{\Gamma(1 / 2)} h^{-1 / 2} e^{-\beta h} I_{(0, \infty)}(h) .
$$

As prior for $h$ we propose $\pi_{\mathrm{bw}}(\cdot \mid \widehat{\beta})$, where $\widehat{\beta}=1.35 /(2 \widehat{R})$ and $\widehat{R}$ is the sample interquartile range. The factor of 1.35 is chosen so that when the data are normally distributed $\widehat{R}$ consistently estimates the population standard deviation, and the $1 / 2$ ensures that the variance of the prior is relatively large. Another obvious choice of prior distribution for $h$ would be an inverse-gamma distribution, since the bandwidth $h$ can be regarded as a scale parameter for the cross-validated likelihood (3). However, after some experimentation we found that prior (6) seems to work better for testing purposes. This is perhaps due to the fact that the inverse-gamma density drastically downweights values of $h$ near 0 , which is where the likelihood will be largest.

As prior for $(\mu, \sigma)$, we propose one of the form

$$
\pi_{0}(\mu, \sigma)=\frac{1}{\sigma} g_{1}\left(\frac{\mu-m}{\sigma}\right) \frac{1}{\gamma} g_{2}\left(\frac{\sigma}{\gamma}\right)
$$

where $g_{1}$ and $g_{2}$ are densities with respective supports the real line and the positive reals, $m$ is a location estimate and $\gamma$ a scale estimate. By choosing the parameters of $(7)$ to be location and scale estimates (and using prior $\pi_{\mathrm{bw}}(\cdot \mid \widehat{\beta})$ for $\left.h\right)$, the resulting ratio of marginal likelihoods will be invariant to location and scale. When the null family is $\mathcal{M}_{0}$, a seemingly reasonable choice for $g_{1}$ would be $f_{0}$, since in that case the prior for $\mu$ given $\sigma$ would contain an amount of information equal to just a single observation from $f_{0}$. An obvious choice for $g_{2}$ would be either a gamma or inverse gamma distribution, with parameters chosen so that the prior is relatively diffuse.

Using prior distributions (6) and (7), the distribution of $\mathrm{MLR}_{n}$ in (4) is invariant to location and scale, as stated in Theorem 1.

Theorem 1. Suppose that $\boldsymbol{X}_{n}=\left(X_{1}, \ldots, X_{n}\right)$ is a random sample from a continuous distribution, $n>2$, and let $\mathcal{M}_{0}$ be as in (5). The following hypotheses are to be tested:

$$
H_{0}: f \in \mathcal{M}_{0} \quad \text { vs. } \quad H_{1}: f \notin \mathcal{M}_{0} .
$$


Suppose that priors of the form (6) and (7) are used for $h$ and $(\mu, \sigma)$, respectively. Furthermore, suppose that $\beta$ is a scale estimate, $m$ a location estimate and $\gamma$ a scale estimate. Then the marginal likelihood ratio (4) is invariant to $\left(\mu_{0}, \sigma_{0}\right)$, the true value of the parameter $(\mu, \sigma)$.

The proof of Theorem 1 is given in the Appendix.

\subsection{Choice of kernel}

In general, the quality of a kernel density estimate is known to depend primarily on the choice of bandwidth $h$, and only secondarily on the choice of kernel (see, e.g., Silverman (1986), and Wand and Jones (1995)). However, the same cannot be said about kernel choice with respect to behavior of the cross-validated nonparametric likelihood we consider in (3). In particular, when the true density is heavy-tailed and the kernel too light-tailed, the estimated likelihood (3) is known to behave poorly (Hall, 1987), resulting in infinite loss and inconsistent estimation. Hall (1987) points out that the kernel $K$ needs to be chosen so that tail effects become negligible. A general rule says that the thicker the tails of the underlying density, the thicker the tails required of the kernel, and the following practical choice was suggested by Hall (1987) :

$$
K_{0}(z)=\left\{(8 \pi e)^{1 / 2} \Phi(1)\right\}^{-1} \exp \left[-\frac{1}{2}\{\log (1+|z|)\}^{2}\right], \quad-\infty<z<\infty,
$$

where $\Phi$ denotes the standard normal distribution function and $\sqrt{8 \pi e} \Phi(1)$ $\doteq 1 / 0.1438$. Note that tails of $K_{0}(z)$ decrease more slowly than $\exp \left(-|z|^{\kappa}\right)$ for any $\kappa>0$ (Hall, 1987). Thus, based on these remarks we anticipate the possibility that our marginal likelihood ratio will, in general, be better behaved when using the kernel $K_{0}$ as opposed to the more traditional Gaussian kernel. This issue will be addressed in simulation studies in the next section.

Another, indispensable, requirement of $K$ and the prior $\pi_{\mathrm{bw}}$ is that they ensure integrability of $\pi_{\mathrm{bw}}(h) L\left(h \mid \boldsymbol{X}_{n}\right)$. The following theorem provides easily checked conditions under which integrability holds.

Theorem 2. Suppose that $\boldsymbol{X}_{n}=\left(X_{1}, \ldots, X_{n}\right)$ is a random sample from a continuous distribution, $n>2$ and that the kernel $K$ satisfies the following conditions:

- $K$ is a bounded, symmetric density that is monotone decreasing on $[0, \infty)$. 
- $\int_{0}^{\infty} y^{r} K^{n}(y) d y<\infty$ for each positive number $r$ and each $n>2$.

If in addition the prior $\pi_{\mathrm{bw}}$ for $h$ is bounded for all $h$ greater than some positive number $a$, and if there exist positive numbers $b$ and $c$ such that $\pi_{\mathrm{bw}}(h)<b h^{-c}$ for $0<h \leq a$, then $\int_{0}^{\infty} \pi_{\mathrm{bw}}(h) L\left(h \mid \boldsymbol{X}_{n}\right) d h<\infty$ almost surely.

It is not difficult to verify that the both the Gaussian kernel and $K_{0}$ satisfy the conditions of Theorem 1 with prior $\pi_{\mathrm{bw}}$ in (6), which means that for these kernels $\pi_{\mathrm{bw}}(h) L\left(h \mid \boldsymbol{X}_{n}\right)$ is integrable. The proof is given in the Appendix.

\subsection{Testing normality and double exponentiality}

In the important special case of testing normality, we use a reference prior for $(\mu, \sigma)$ of the form (7). Specifically, $g_{1}$ is a standard normal density,

$$
g_{2}(s)=\frac{2}{\sqrt{\pi}} \cdot \frac{1}{s^{2}} e^{-1 / s^{2}} I_{(0, \infty)}(s),
$$

$m$ is the sample mean and $\gamma=\widehat{\sigma} / \sqrt{2}$, where $\widehat{\sigma}$ is the sample standard deviation. Then, the denominator of $\mathrm{MLR}_{n}$ becomes

$$
\begin{aligned}
m_{0}^{N} & =\int_{\Theta} \prod_{i=1}^{n} f_{0}\left(X_{i} \mid \boldsymbol{\theta}\right) \pi_{0}(\boldsymbol{\theta}) d \boldsymbol{\theta} \\
& =\Gamma\left(\frac{n+1}{2}\right)(n+1)^{-(n+2) / 2} \pi^{-(n+1) / 2} \widehat{\sigma}^{-n}
\end{aligned}
$$

and the corresponding $\mathrm{MLR}_{n}$ is

$$
\operatorname{MLR}_{n}=\frac{\int_{0}^{\infty} \pi_{\mathrm{bw}}(h) L\left(h \mid \boldsymbol{X}_{n}\right) d h}{m_{0}^{N}} .
$$

Additionally, we consider testing the null hypothesis that the data come from a double exponential distribution, denoted $\mathrm{DE}(\mu, \sigma)$. This is a locationscale family with density

$$
f(x \mid \mu, \sigma)=\frac{1}{2 \sigma} \exp \left(-\frac{1}{\sigma}|x-\mu|\right),
$$

where $\mu$ is the median (mean) of $X$ and $\sigma$ the scale parameter. Again we use a reference prior for $(\mu, \sigma)$ as in (7). Specifically, $g_{1}$ is a double exponential 
density with $\mu=0, \sigma=1$, and $m$ the sample median $\widehat{m}$. For $g_{2}$, we use an inverse gamma density with $\alpha=1$ and $\beta=1$. Then,

$$
\pi_{0}(\mu, \sigma) \propto \frac{1}{2 \sigma} \exp \left(-\frac{1}{\sigma}|\mu-\widehat{m}|\right) \times \frac{1}{\gamma}(\sigma / \gamma)^{-2} \exp \left(-\frac{\gamma}{\sigma}\right) .
$$

Note that the mode of the prior for $\sigma$ is $\gamma / 2$. Thus, to make the prior centered at a mode estimate $\widehat{\sigma}$ of $\sigma$, we may take $\widehat{\gamma}=\widehat{R} / \log 2$, since the interquartile range of $X \sim \mathrm{DE}(\mu, \sigma)$ is $2 \sigma \log 2$.

With the above choice of prior, the marginal likelihood under the null hypothesis of double exponentiality is

$$
\begin{aligned}
m_{0}^{D E} & =\int_{\Theta} \prod_{i=1}^{n} f_{0}\left(X_{i} \mid \boldsymbol{\theta}\right) \pi_{0}(\boldsymbol{\theta}) d \boldsymbol{\theta} \\
& =\left(\frac{1}{2}\right)^{n+1}(n+1) ! \widehat{\gamma} \int_{-\infty}^{\infty}\left[\sum_{i=1}^{n}\left|X_{i}-\mu\right|+|\mu-\widehat{m}|+\widehat{\gamma}\right]^{-(n+2)} d \mu
\end{aligned}
$$

and the corresponding $\mathrm{MLR}_{n}$ is

$$
\operatorname{MLR}_{n}=\frac{\int_{0}^{\infty} \pi_{\mathrm{bw}}(h) L\left(h \mid \boldsymbol{X}_{n}\right) d h}{m_{0 n}^{D}} .
$$

The integral in the marginal likelihood $m_{0}^{D E}$ can be computed exactly, and details are given in the Appendix. Hence, in the computation of the marginal likelihood ratio $\mathrm{MLR}_{n}$, either (9) or (12), the only remaining problem is evaluating the kernel-based marginal likelihood. This can be achieved by means of numerical integration, using, for example, the built-in $\mathrm{R}$ function integrate(), as we do in obtaining the results in the next section.

\section{Numerical results}

In this section we use simulation to investigate power properties of our test procedure based on the marginal likelihood ratio, $\mathrm{MLR}_{n}$, and also apply our test to data from an astronomical setting. In addition to the ShapiroWilk and Kolomogorov-Smirnov tests in the respective cases of testing normality and double exponentiality, we consider a goodness-of-fit test of normality that was recently proposed by Vexler and Gurevich (2010). Their test uses empirical likelihood ratios and is implemented with the $\mathrm{R}$ package dbEmpLikeGOF (Miecznikowski et al., 2013). Our software implementation of the proposed goodness-of-fit test with $\mathrm{MLR}_{n}$ is written in $\mathrm{R}$, which is available from the authors upon request. 


\subsection{Testing normality against mixture alternatives}

Here we test the null hypothesis of normality using an $\alpha$ of 0.05 . We consider sample sizes $n=10,11, \ldots, 400$, and at each sample size $n, 100$ data sets were drawn from nonnormal densities. In doing so, a somewhat nonstandard method was used so as to diversify the collection of alternatives considered. Each time a data set is generated, a density is randomly selected from a family of densities as described below, and then a sample of size $n$ is selected from the randomly chosen density.

Each density generated is a random mixture of two or more normals, having the form

$$
f(x)=\sum_{i=1}^{M} w_{i} \frac{1}{\sigma_{i}} \phi\left(\frac{x-\mu_{i}}{\sigma_{i}}\right) .
$$

Densities are generated as follows:

- A value of $M$ between 2 and 20 is selected from a distribution such that the probability of $m$ is proportional to $m^{-1}, m=2, \ldots, 20$.

- Given $M=m$, values $w_{1}, \ldots, w_{m}$ are selected from the Dirichlet distribution with all $m$ parameters equal to $1 / 2$.

- Given $m$ and independent of $w_{1}, \ldots, w_{m}, 1 / \sigma_{1}^{2}, \ldots, 1 / \sigma_{m}^{2}$ are independent and identically distributed as gamma with shape and rate each $1 / 2$, and, conditional on $\sigma_{1}, \ldots, \sigma_{m}, \mu_{1}, \ldots, \mu_{m}$ are independent with $\mu_{j}$ distributed $N\left(0, \sigma_{j}^{2}\right), j=1, \ldots, m$.

Generating densities in this way provides a variety of different distributional types, including ones that are skewed, lepto- or platykurtic, and/or multimodal (see, e.g., Marron and Wand (1992)).

Four tests were considered: the Shapiro-Wilk (SW), the empirical likelihood ratio goodness of fit test of Vexler and Gurevich (2010) denoted as ELRT, and two marginal likelihood ratio tests, one using a Gaussian kernel and the other kernel $K_{0}$. The latter two tests will be referred to as $\operatorname{MLRT}(\phi)$ and $\operatorname{MLRT}\left(K_{0}\right)$, respectively. Critical values for $\operatorname{MLRT}(\phi)$ and $\operatorname{MLRT}\left(K_{0}\right)$ were obtained by means of simulation.

Results are summarized in Figure 1. The Shapiro-Wilk test is slightly superior to $\operatorname{MLRT}\left(K_{0}\right)$, which in turn is superior to $\operatorname{MLRT}(\phi)$, whereas ELRT is inferior to the Shapiro-Wilk test and $\operatorname{MLRT}\left(K_{0}\right)$ but comparable to $\operatorname{MLRT}(\phi)$. The superiority of $K_{0}$ pointed out by Hall (1987) in the context of bandwidth selection appears to carry over to this particular testing context for the random mixture of normal distributions. 


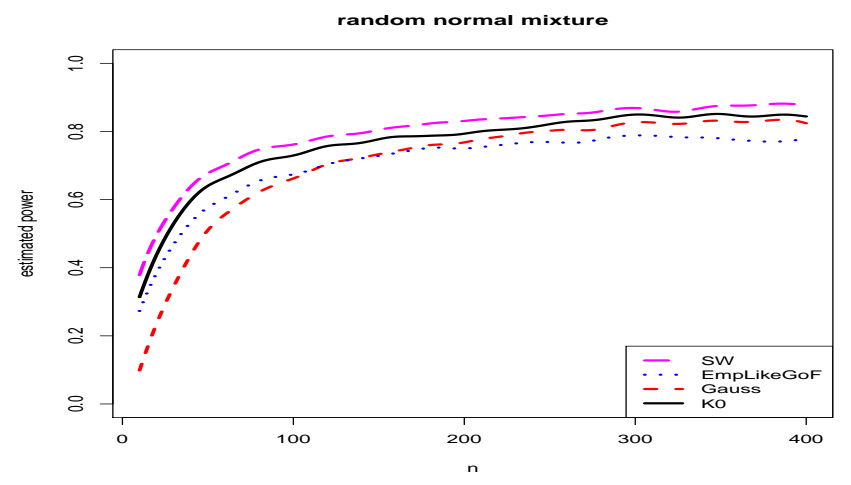

Figure 1: Estimated power curves when testing normality at level 0.05. Each curve is a local linear estimate fitted from empirical powers for $n=10, \ldots, 400$. The number of replications at each $n$ was 100 . The solid line corresponds to $\operatorname{MLRT}\left(K_{0}\right)$, the dashed line to $\operatorname{MLRT}(\phi)$, the long-dashed line to the Shapiro-Wilk test and the dotted line to the ELRT.

\subsection{Testing normality against short-tailed and heavy-tailed alternatives}

Here we test the null hypothesis of normality when the alternative is either short- or heavy-tailed. As short-tailed alternatives we consider a uniform density on $(-1,1)(U(-1,1))$ and a symmetric triangular density on $(-1,1)(\operatorname{Tri}(-1,1))$. Note that the chosen support of these densities does not affect power since our tests are invariant to location and scale. Let $t(r)$ denote the $t$-distribution with $r$ degrees of freedom. As heavytailed alternatives we consider $t(1)$, the Cauchy distribution, and $t(2)$. At each sample size $n=10,11, \ldots, 400,100$ data sets were drawn from each of $U(-1,1)$, Tri $(-1,1), t(1)$ and $t(2)$. The same four tests as before are applied to each data set generated.

Results are summarized in Figure 2. In contrast to the normal mixture alternatives in Section 4.1, for the short-tailed alternatives, $U(-1,1)$ and Tri $(-1,1)$, the Shapiro-Wilk test no longer dominates the two MLRT tests. Indeed, $\operatorname{MLRT}(\phi)$ and ELRT are considerably more powerful than the Shapiro-Wilk test, while $\operatorname{MLRT}\left(K_{0}\right)$ has power similar to that of ShapiroWilk. The fact that $\operatorname{MLRT}(\phi)$ performs well in this case is not too surprising since likelihood cross-validation with a Gaussian kernel performs much better for short than heavier tailed densities. On the other hand, for the heavy-tailed alternatives, Cauchy and $t(2)$, the Shapiro-Wilk test is slightly superior to $\operatorname{MLRT}\left(K_{0}\right)$, which in turn is superior to $\operatorname{MLRT}(\phi)$. Also, ELRT is inferior to the Shapiro-Wilk test and $\operatorname{MLRT}\left(K_{0}\right)$, which is similar to the results for normal mixture alternatives in Section 4.1. 

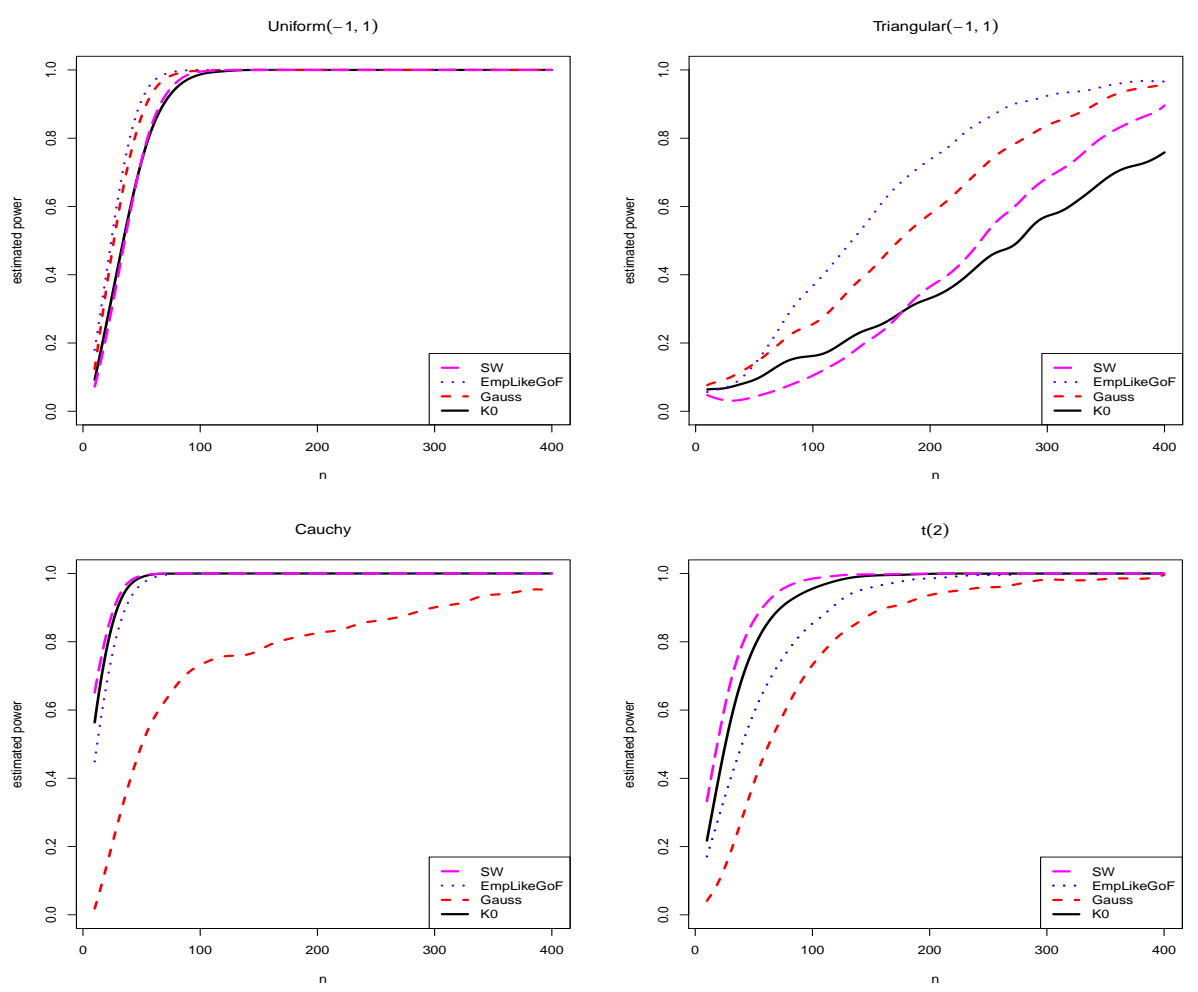

Figure 2: Estimated power curves when testing normality at level 0.05. Each curve is a local linear estimate fitted from empirical powers for $n=10, \ldots, 400$. The number of replications at each $n$ was 100 . The solid line corresponds to $\operatorname{MLRT}\left(K_{0}\right)$, the dashed line to $\operatorname{MLRT}(\phi)$, the long-dashed line to the Shapiro-Wilk test and the dotted line to the ELRT.

\subsection{Testing double exponentiality}

We now consider testing the null hypothesis that the data come from some double exponential distribution. When testing the fit of a nonnormal distribution, there is often no analog of the Shapiro-Wilk test, in which case the nonparametric goodness-of-fit test of choice is most likely one based on the empirical cumulative distribution function, such as the KolmogorovSmirnov or Anderson-Darling tests.

We follow the same simulation setup as in Sections 4.1 and $4.2 ; \alpha$ is 0.05 , and at each sample size $n, n=10,11, \ldots, 400,100$ data sets are drawn from densities that are not double-exponentially distributed. We consider three cases, one with alternatives generated from double exponential (DE) 
mixtures, one with short-tailed alternatives and one with heavy-tailed alternatives. In order to generate the DE mixtures, we use the same random selection procedure described in Section 4.1. Each time a data set is generated, a density is randomly selected from a mixture of two or more DE's, having the form

$$
f(x)=\sum_{i=1}^{M} w_{i} \frac{1}{\sigma_{i}} f_{\mathrm{DE}}\left(\frac{x-\mu_{i}}{\sigma_{i}}\right),
$$

where $f_{\mathrm{DE}}$ denotes the probability density function of $\mathrm{DE}(0,1)$.

Three tests were considered: the Kolmogorov-Smirnov (KS), MLRT $(\phi)$ and $\operatorname{MLRT}\left(K_{0}\right)$. Critical values for all three tests were obtained by simulation, since, to our knowledge, critical values for the KS test are not known when testing double exponentiality. Results are summarized in Figure 3.

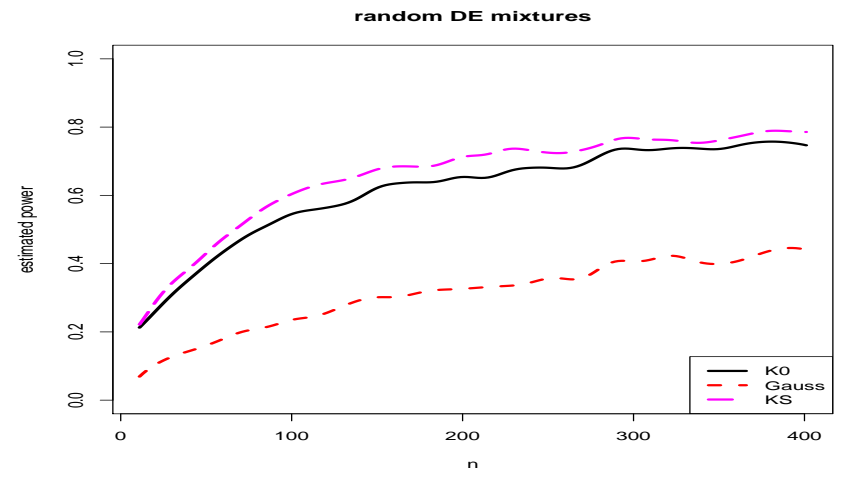

Figure 3: Estimated power curves when testing double exponentiality at level 0.05. Each curve is a local linear estimate fitted from empirical powers for $n=10, \ldots, 400$. The number of replications at each $n$ was 100 . The solid line corresponds to $\operatorname{MLRT}\left(K_{0}\right)$, the dashed line to $\operatorname{MLRT}(\phi)$ and the long dashed line to the Kolmogorov-Smirnov test.

The KS test is slightly superior to $\operatorname{MLRT}\left(K_{0}\right)$, which in turn is superior to $\operatorname{MLRT}(\phi)$. This is analogous to our results when testing normality against random mixtures, where the Shapiro-Wilk test was used instead of KS. In the double exponential case the superiority of $K_{0}$ over $\phi$ is more pronounced than it was when testing normality. Again, this fits with the results of Hall (1987) in the context of bandwidth selection, wherein the Gaussian kernel does not perform well when the underlying density is heavier tailed.

We also consider power when testing double exponentiality against the same short- and heavy-tailed alternatives as in Section 3.2. Results are summarized in Figure 4, where we see a somewhat different pattern than 
that observed when testing normality. When testing double exponentiality for the short-tailed alternatives, our two proposed tests behave similarly to each other, whereas $\operatorname{MLRT}(\phi)$ was clearly better than $\operatorname{MLRT}\left(K_{0}\right)$ when testing normality. Furthermore, both $\operatorname{MLRT}(\phi)$ and $\operatorname{MLRT}\left(K_{0}\right)$ are far superior to the KS test when testing double exponentiality. When testing double exponentiality against the heavy-tailed alternatives, $\operatorname{MLRT}\left(K_{0}\right)$ was considerably better than KS and $\operatorname{MLRT}(\phi)$, as evidenced Figure 4.
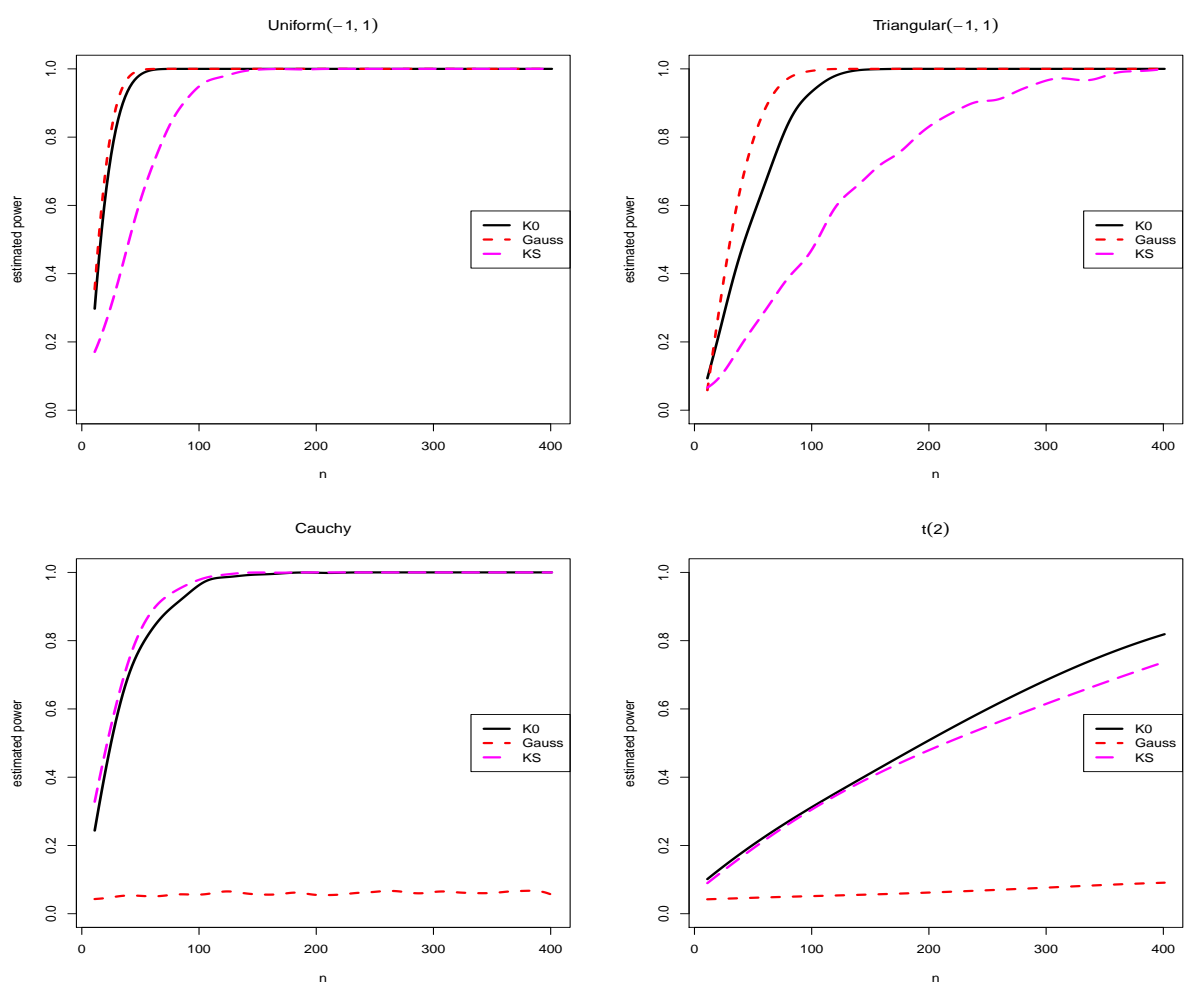

Figure 4: Estimated power curves when testing normality at level 0.05. Each curve is a local linear estimate fitted from empirical powers for $n=10, \ldots, 400$. The number of replications at each $n$ was 100 . The solid line corresponds to $\operatorname{MLRT}\left(K_{0}\right)$, the dashed line to $\operatorname{MLRT}(\phi)$, the long-dashed line to the Kolmogorov-Smirnov test.

Note that no one test procedure dominates all others in our power analysis. The proposed tests $\operatorname{MLRT}\left(K_{0}\right)$ and $\operatorname{MLRT}\left(\phi_{0}\right)$ are adequate alternatives to SW and KS for heavy-tailed distributions and more powerful than SW and KS for short-tailed distributions. As the sample size increases, all the tests are indistinguishable in terms of power. Also, notice that the new tests were more powerful in testing normality than the recently proposed test of 
Vexler and Gurevich (2010), which itself is not always better than SW and KS, either in our study or that of Vexler and Gurevich (2010).

\subsection{Analysis of planetary nebula luminosity data}

As a real application, we analyze observations of planetary nebula luminosity in the Messier 31, or Andromeda, galaxy. For background on the data analyzed, the reader is referred to Ciardullo et al. (1989) and Ciardullo et al. (2002). The frequency distribution of planetary nebula luminosities is referred to as a planetary nebula luminosity function, or PNLF. From a visual inspection of a histogram of Messier 31 data, Ciardullo et al. (1989) claimed that the PNLF of Messier 31 does not follow a power law, also known as the Pareto distribution. We wish to validate this claim using the test we have proposed.

The available data from Messier 31 are brightness readings from 238 planetary nebulae, which can be downloaded from http://astrostatistics . psu.edu/datasets/plan_neb.html. These readings are measured on a scale such that smaller values correspond to brighter objects. Ciardullo et al. (1989) note that dimmer readings are subject to more measurement error. Therefore, they used only observations smaller than 22 to fit the PNLF curve.

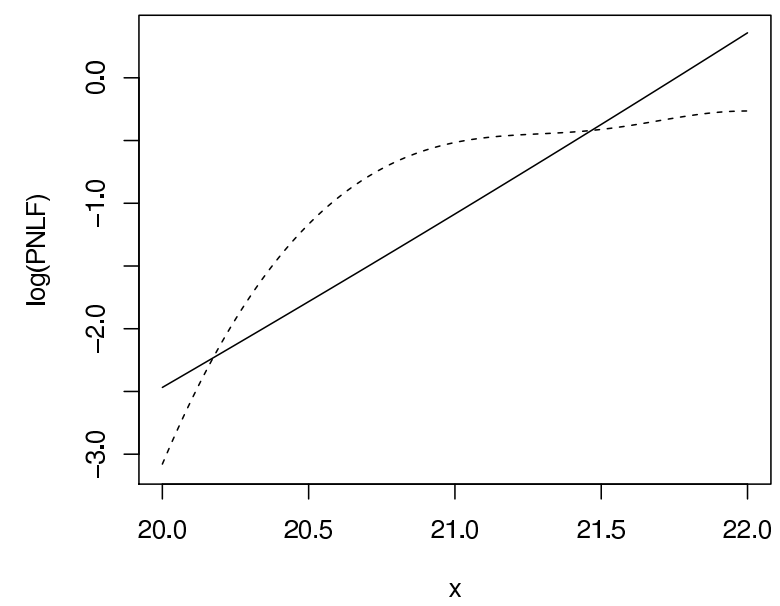

Figure 5: Estimates of log-density of luminosity. The solid line is a fitted power law curve that maximizes the posterior density. The dashed line is the log of a kernel density estimate. Each estimate is computed from 61 luminosity readings smaller than 22 .

In statistical parlance, their data are censored. Let $X_{1}, \ldots, X_{n}$ be the actual observations and $Y_{1}, \ldots, Y_{n}$ be the underlying data that are free of 
errors. When $X_{i}<22$, it is assumed that $X_{i}=Y_{i}$, but if $X_{i}>22$ then we only know that $Y_{i}>22$. We wish to test whether the PNLF curve follows a power law at brightnesses less than 22. Plots of kernel and power law estimates of log-density are shown in Figure 5. Of the 238 observed luminosities, only 61 were smaller than 22 .

The null density of $Y_{i}$ is such that

$$
f_{0}(y)=(1-p) f(y \mid \alpha), \quad y<22,
$$

where $p=P\left(Y_{i}>22\right)$ and

$$
f(y \mid \alpha)=\frac{\alpha 22^{\alpha}}{(44-y)^{\alpha+1}} I_{(-\infty, 22)}(y) .
$$

The parameters $p$ and $\alpha$ are unknown, with $\alpha>0$. Under the null hypothesis, the likelihood function is

$$
L(\alpha, p)=p^{n_{C}}(1-p)^{n-n_{C}} \prod_{i \in S} f\left(x_{i} \mid \alpha\right),
$$

where $n_{C}$ is the number of observations larger than 22 and $S$ is the set of indices $i$ such that $x_{i}<22$. Likewise, the kernel likelihood has the form

$$
L_{K}(h, p)=p^{n_{C}}(1-p)^{n-n_{C}} \prod_{i \in S} \widehat{f}_{i}\left(x_{i} \mid h\right),
$$

where the kernel estimates are based only on observations smaller than 22 .

If $p$ is assumed a priori independent of $h$ and $\alpha$, then the marginal likelihood ratio is simply

$$
\operatorname{MLR}_{n}=\left[\int_{0}^{\infty} \pi(h) \prod_{i \in S} \widehat{f}_{i}\left(x_{i} \mid h\right) d h\right]\left[\int_{0}^{\infty} \pi_{0}(\alpha) \prod_{i \in S} f\left(x_{i} \mid \alpha\right) d \alpha\right]^{-1} .
$$

In other words, the marginal likelihood ratio has exactly the same form it would have if the uncensored data were a random sample of size $n-n_{C}$ from density $(1-p)^{-1} f(y) I_{(-\infty, 22)}(y)$, where $f$ is the true density of $Y_{i}$. To compute $\mathrm{MLR}_{n}$ in (16) we assumed that the prior for $h$ is $\pi(\cdot \mid \widehat{\beta})$, as defined in Section 2.1. We used a gamma prior $\pi_{0}(\alpha)$ for $\alpha$, which is a conjugate prior in this case, taking the shape parameter to be 2 and the rate parameter equal to $M=\sum_{i \in S} \log \left(\left(44-x_{i}\right) / 22\right) /\left(n-n_{C}\right)$. This prior has an amount of information equivalent to one observation from the power law density.

As is well-known, kernel estimates are subject to edge effects when the underlying density does not tend to 0 at the endpoints of its support. Such 
is the case here at $x=22$ because of the data censoring. We thus used a reflection technique (Silverman, 1986, p.30) to compute each $\widehat{f}_{i}\left(x_{i} \mid h\right)$ in the kernel likelihood. This involves defining data $x_{i}^{*}=44-x_{i}, i \in S$, which lie to the right of the truncation point 22 , and then computing density estimates using the enlarged data set of $2\left(n-n_{C}\right)$ values. More precisely, we have

$$
\widehat{f}_{i}\left(x_{i} \mid h\right)=\frac{1}{(n-1) h} \sum_{j \in S, j \neq i}\left\{K\left(\frac{x_{i}-x_{j}}{h}\right)+K\left(\frac{x_{i}-x_{j}^{*}}{h}\right)\right\} .
$$

Using $(n-1)$ in the last expression rather than $2(n-1)$ ensures that the kernel estimate integrates to 1 on $(-\infty, 22)$.

The value of $\mathrm{MLR}_{n}$ using the priors and density estimates discussed above was 1374 . To evaluate the value of $\mathrm{MLR}_{n}$, we must simulate values of $\mathrm{MLR}_{n}$ under the null hypothesis for approximating its null distribution. Because $\alpha$ is neither a location nor a scale parameter, the distribution of $\mathrm{MLR}_{n}$ depends on the unknown value of $\alpha$. The most common way of dealing with this problem is to draw many samples from $f(\cdot \mid \widehat{\alpha})$, where $\widehat{\alpha}$ is a point estimate of $\alpha$ computed from the original set of data. The MLE of $\alpha$ is $\sum_{i=1}^{n} \log \left(\left(44-y_{i}\right) / 22\right)$, whose value for the Messier 31 luminosity data is 31.48. We generated 10,000 samples of size 61 from the power law density $f(\cdot \mid 31.48)$ and computed $\mathrm{MLR}_{n}$ for each sample. The resulting 95th percentile of the empirical distribution was 3.38, and only 4 of 10,000 simulated values were larger than 1374 , leading to an approximate $P$-value of 0.0004 .

Let $q(\alpha)$ be the 95 th percentile of the distribution of $\mathrm{MLR}_{n}$ when $H_{0}$ is true and $\alpha$ is the true parameter value. Ideally we would compare $\mathrm{MLR}_{n}$ with $q\left(\alpha_{0}\right)$, where $\alpha_{0}$ is the correct value of $\alpha$. Since $\hat{\alpha}$ is at least somewhat different than $\alpha_{0}$, it is reasonable to ask how much $q(\alpha)$ varies throughout an interval of likely values for $\alpha_{0}$. The asymptotic variance of the maximum likelihood estimator of $\alpha$ in the power law density is $\alpha^{2} / n$, which is the reciprocal of the information in a random sample of size $n$. Hence an approximate $95 \%$ confidence interval for $\alpha_{0}$ is $31.48 \pm 1.96(31.48 / \sqrt{61})$. We may approximate $q(\alpha)$ over this interval of $\alpha$ values by means of simulation, using the following algorithm.

1. Generate a value of $\alpha$, call it $\alpha^{*}$, from a normal distribution with mean 31.48 and standard deviation $31.48 / \sqrt{61}=4.03$.

2. Generate a random sample of size 61 from the power law density $f\left(\cdot \mid \alpha^{*}\right)$. 
3. Compute a marginal likelihood ratio $\mathrm{MLR}_{n}^{*}$ from the data in step 2 in exactly the same way that $\mathrm{MLR}_{n}$ was computed from the original data.

The previous three steps were repeated independently 100,000 times, and Figure 6 shows a scatterplot of the $100,000\left(\alpha, \log \left(\mathrm{MLR}_{n}\right)\right)$ pairs. The line is a near neighbor type estimate of the function $q(\alpha)$. At a given $\alpha$, say $x$, the estimate is the 95 th percentile of the 1000 values of $\log \left(\mathrm{MLR}_{n}\right)$ whose corresponding $\alpha$ s are nearest $x$. These results provide strong evidence that $q(\alpha)$ varies little throughout the interval $(15,45)$. Coupled with the previous bootstrap results at $\hat{\alpha}=31.48$, we feel there is strong evidence for rejecting the hypothesis that the PNLF curve is a power law density.

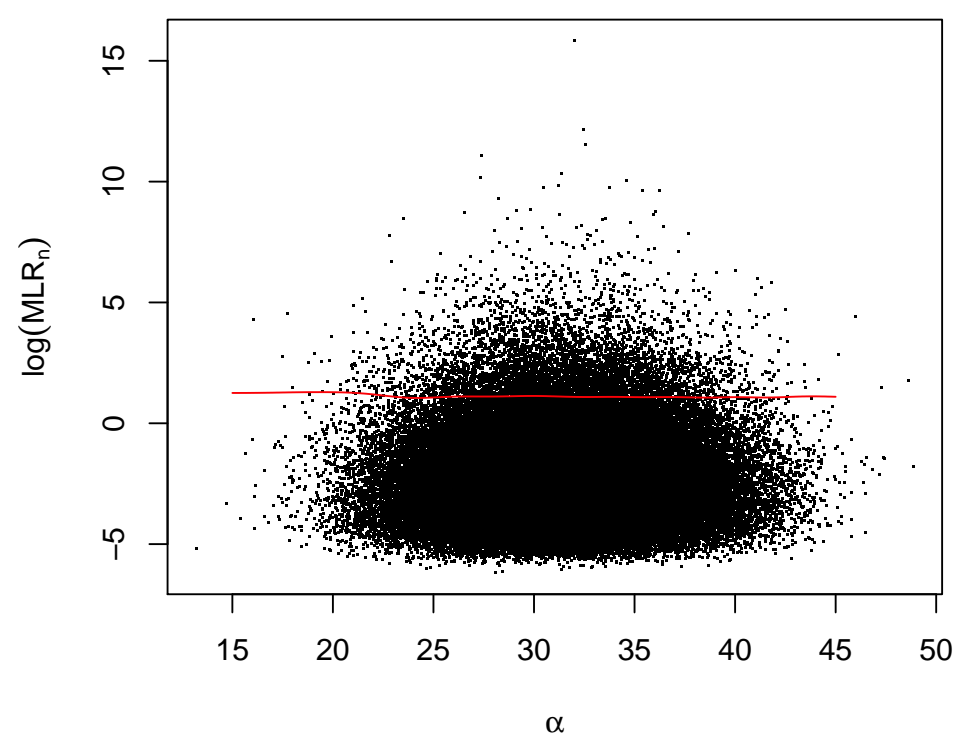

Figure 6: Scatterplot of $\log \left(\mathrm{MLR}_{n}\right)$ versus $\alpha$ for data from bootstrap simulation. The line is an estimate of the 95 th percentile of the distribution of $\log \left(\mathrm{MLR}_{n}\right)$ given $\alpha$.

\section{Multivariate goodness of fit}

Our marginlal likelihood ratio test, MLRT is easily generalized to the multivariate setting. Suppose, for example, that one wishes to test the fit of 
a parametric model for bivariate data. Let $\boldsymbol{X}_{i}^{T}=\left(X_{i 1}, X_{i 2}\right)$, and suppose that $\boldsymbol{X}_{1}, \ldots, \boldsymbol{X}_{n}$ are independent and identically distributed observations with common density $f$. A kernel estimate of $f(\boldsymbol{x})$ is

$$
\widehat{f}(\boldsymbol{x} \mid H)=\frac{1}{n|H|} \sum_{i=1}^{n} K\left(H^{-1}\left(\boldsymbol{x}-\boldsymbol{X}_{i}\right)\right),
$$

where $H$ is a two by two bandwidth matrix. (The reader is referred to Scott (1992) for a comprehensive discussion of multivariate kernel density estimation.) A common simplification is to let $H$ be diagonal with positive elements $h_{1}$ and $h_{2}$ on the diagonal. It is also common to define $K$ to be a product of univariate kernels. We thus suggest using a kernel estimate $\widehat{f}\left(\cdot \mid h_{1}, h_{2}\right)$ in which $H=\operatorname{diag}\left(h_{1}, h_{2}\right)$ and

$$
K\left(u_{1}, u_{2}\right)=K_{0}\left(u_{1}\right) K_{0}\left(u_{2}\right),
$$

where use of $K_{0}$ is proposed based on its success in the univariate case. The marginal corresponding to the kernel estimate would be

$$
\int_{0}^{\infty} \int_{0}^{\infty} L\left(h_{1}, h_{2}\right) \pi\left(h_{1}, h_{2}\right) d h_{1} d h_{2}
$$

where $L$ is a leave-one-out likelihood defined as in the univariate case and $\pi\left(h_{1}, h_{2}\right)$ is the prior for $\left(h_{1}, h_{2}\right)$.

Consider the important case of testing for bivariate normality. As in the univariate case we desire that the marginal likelihood ratio $\mathrm{MLR}_{n}$ be invariant to location and scale. This is accomplished by choosing priors as follows. Take $\pi\left(h_{1}, h_{2}\right)=\pi_{\mathrm{bw}}\left(h_{1} \mid \widehat{\beta}_{1}\right) \pi_{\mathrm{bw}}\left(h_{2} \mid \widehat{\beta}_{2}\right)$, where, $\pi_{\mathrm{bw}}$ is the prior (6) used for $h$ in the univariate problem. As before, $\widehat{\beta}_{j}=(1 / 2) /\left(1.35 \widehat{R}_{j}\right)$, $j=1,2$, where $\widehat{R}_{j}$ is the sample interquartile range of $X_{1 j}, \ldots, X_{n j}$.

It is worth noting that Zhang et al. (2009) and Zhang et al. (2006) have also proposed priors for bandwidth matrices. Now let $\boldsymbol{\mu}$ and $\boldsymbol{\Sigma}$ be the mean and covariance matrix, respectively, of the posited bivariate normal distribution, and as prior for $\boldsymbol{\Sigma}$ use an inverse-Wishart distribution with 2 degrees of freedom and scale matrix the inverse of $\boldsymbol{S}^{2}$, the sample covariance matrix. The prior of $\boldsymbol{\mu}$ given $\boldsymbol{\Sigma}$ is bivariate normal with mean equal to the sample mean vector and covariance matrix $\boldsymbol{\Sigma}$. This prior for $(\boldsymbol{\mu}, \boldsymbol{\Sigma})$ is centered at the sample mean and sample covariance matrix, but contains minimal information about the parameters.

While the null distribution of the marginal likelihood ratio $\mathrm{MLR}_{n}$ defined in the last paragraph is invariant to location and scale, it is not invariant to 
$\rho$, the correlation between $X_{i 1}$ and $X_{i 2}$. To deal with this problem in practice one may use a parametric bootstrap, similar to the bootstrap algorithm described in Section 3.5. Let $\widehat{\rho}$ be the (Pearson) sample correlation coefficient for the data $\boldsymbol{X}_{1}, \ldots, \boldsymbol{X}_{n}$. Then we may generate independent random samples of size $n$ from a bivariate normal distribution with mean vector $(0,0)$, unit variances and correlation $\widehat{\rho}$. The marginal likelihood ratio is computed for each bootstrap sample, and critical values are approximated by sample percentiles of all the marginal likelihood ratios. This procedure will result in an asymptotically valid test as $n$ tends to $\infty$ since $\widehat{\rho}$ is a $\sqrt{n}$-consistent estimator of $\rho$.

As emphasized before, an advantage of our approach with the marginal likelihood ratio using the kernel estimates in both the univariate and multivariate cases is its relative computational simplicity. MCMC methods are not required to compute the test statistic, i.e., the marginal likelihood ratio, assuming anyway that the marginal of the parametric model can be computed analytically. Even if the marginal cannot be determined exactly, one may use a Laplace approximation for it.

\section{Conclusion}

We have proposed a nonparametric procedure for testing the goodness of fit of a parametric model. The test statistic is based on a nonparametric marginal likelihood ratio in which the two marginals correspond to a kernel estimate and the parametric model. This statistic has the form of a Bayes factor, but the test itself is carried out in frequentist fashion. The frequentist method closest to our approach would be a likelihood ratio test (LRT). Rather than using the ratio of maximized likelihoods (as in an LRT), the new statistic uses the ratio of integrated, weighted likelihoods, where the weights are obtained from prior densities. By using appropriate data-tuned priors for unknown parameters, it was shown that the distribution of the proposed test statistic is invariant to location and scale. Our proposed test is performed by comparing the marginal likelihood ratio with its null percentiles. Monte Carlo was used to compare the power of the new tests with that of existing goodness-of-fit procedures when testing either normality or double exponentiality. In a simulation study, the best of our proposed tests was either comparable to or more powerful than the popular Shapiro-Wilk and Kolmogorov-Smirnov tests. Overall, the test based on the kernel $K_{0}$ of Hall (1987) was more powerful than the one based on a Gaussian kernel. In particular this is true when the alternative density is long-tailed. 
The proposed testing procedure is easily applicable in practice for testing the fit of any location and scale family. In comparison to standard Bayesian methods for conducting a nonparametric goodness-of-fit test, our test is quite simple. For the alternative model, only a univariate prior for the bandwidth is required, as opposed to nonparametric priors such as Dirichlet, Polya or Gaussian processes. The marginal likelihood corresponding to the kernel estimate must be evaluated numerically, but this is easily done using the built-in $\mathrm{R}$ function integrate.

Additionally our test was used for testing the fit of a Pareto distribution to verify a claim from the astronomy literature that the planetary nebula luminosity function for the Andromeda galaxy does not follow a power law. Important and challenging questions concern asymptotic properties of the nonparametric marginal likelihood ratio $\mathrm{MLR}_{n}$. Deriving the asymptotic null distribution $\mathrm{MLR}_{n}$ or its asymptotic power against local alternatives are beyond the scope of our current research.

Generalizations of our nonparametric test to multivariate data were discussed in Section 4. Other generalizations are possible, including lack-of-fit testing in regression problems. In regression, a marginal likelihood ratio would require a nonparametric estimate of the mean function. This estimate could be supplied by any of a number of smoothing methods, such as local polynomial regression (Fan and Gijbels, 1996) or orthogonal series (see, e.g., Hart (1997) and Efromovich (1999)).

\section{Acknowledgements}

Research of Jeffrey D. Hart was supported by NSF grant DMS-0604801. Research of Taeryon Choi was supported by Basic Science Research Program through the National Research Foundation of Korea (NRF) funded by the Ministry of Science, ICT \& Future Planning (No. 2013R1A1A2074463). We greatly appreciate all the comments and suggestions from the Associate Editor and the two referees.

\section{Appendix A. Proof of Theorem 1}

First we assume that $m$ and $\gamma$ are location and scale estimates, respectively, with the property that each of $\tilde{m}=\left(m-\mu_{0}\right) / \sigma_{0}$ and $\tilde{\gamma}=\gamma / \sigma_{0}$ is location-scale invariant. The marginal for the parametric model is

$$
\int_{-\infty}^{\infty} \int_{0}^{\infty} \sigma^{-n} \prod_{i=1}^{n} f_{0}\left(\frac{X_{i}-\mu}{\sigma}\right) \sigma^{-1} g_{1}\left(\frac{\mu-m}{\sigma}\right) \gamma^{-1} g_{2}\left(\frac{\sigma}{\gamma}\right) d \sigma d \mu,
$$


and upon making the change of variables $\alpha=\left(\mu-\mu_{0}\right) / \sigma_{0}$ and $\beta=\sigma / \sigma_{0}$, this integral becomes

$$
\sigma_{0}^{-n} \int_{-\infty}^{\infty} \int_{0}^{\infty} \beta^{-n} \prod_{i=1}^{n} f_{0}\left(\frac{Y_{i}-\alpha}{\beta}\right) \beta^{-1} g_{1}\left(\frac{\alpha-\tilde{m}}{\beta}\right) \tilde{\gamma}^{-1} g_{2}\left(\frac{\beta}{\tilde{\gamma}}\right) d \beta d \alpha,
$$

where $Y_{i}=\left(X_{i}-\mu_{0}\right) / \sigma_{0}, i=1, \ldots, n$. It follows that the distribution of the marginal depends on $\left(\mu_{0}, \sigma_{0}\right)$ only through the multiplier $\sigma_{0}^{-n}$.

The marginal based on the kernel estimate is

$$
(n-1)^{-n} \int_{0}^{\infty} h^{-n}\left[\prod_{i=1}^{n} \sum_{j \neq i} K\left(\frac{X_{i}-X_{j}}{h}\right)\right] \pi(h \mid \widehat{\beta}) d h .
$$

Making the change of variable $b=h / \sigma_{0}$, this integral is

$$
\sigma_{0}^{-n}(n-1)^{-n} \int_{0}^{\infty} b^{-n}\left[\prod_{i=1}^{n} \sum_{j \neq i} K\left(\frac{Y_{i}-Y_{j}}{b}\right)\right] \sigma_{0} \pi\left(b \sigma_{0} \mid \widehat{\beta}\right) d b .
$$

By the way in which $\pi(\cdot \mid \widehat{\beta})$ is defined, the $\sigma_{0} \pi\left(\sigma_{0} b \mid \widehat{\beta}\right)$ is free of location and scale for each $b$. The location-scale invariance of $\mathrm{MLR}_{n}$ follows from the fact that the term $\sigma_{0}^{-n}$ is common to both numerator and denominator.

\section{Appendix B. Proof of Theorem 2}

By the assumptions $n>2, K$ is bounded, and $\pi_{\mathrm{bw}}(h)$ is bounded for all $h>a$,

$$
\int_{a}^{\infty} \pi_{\mathrm{bw}}(h) L\left(h \mid \boldsymbol{X}_{n}\right) d h<\infty
$$

since the integrand is bounded by a constant times $h^{-n}$. Now, let $\delta_{n}=$ $\min _{i \neq j}\left|X_{i}-X_{j}\right|$, and note that $\delta_{n}$ is almost surely positive, since the distribution of $X_{i}$ is continuous. By assumption

$$
\begin{aligned}
\int_{0}^{a} \pi_{\mathrm{bw}}(h) L\left(h \mid \boldsymbol{X}_{n}\right) d h & <b \int_{0}^{a} h^{-(n+c)} K^{n}\left(\frac{\delta_{n}}{h}\right) d h \\
& =\delta_{n}^{-n-c+1} \int_{\delta_{n} / a}^{\infty} y^{n+c-2} K^{n}(y) d y .
\end{aligned}
$$

By assumption the last integral is finite, and so the proof is complete. 


\section{Appendix C. Marginal likelihood $m_{0}^{D E}$}

Here we derive the integral (11) in the marginal likelihood $m_{0}^{D E}$. For simplicity, assuming that $n$ is odd, the integral (11) may be written as the sum of three terms:

$$
\begin{aligned}
& \int_{-\infty}^{\infty}\left[\sum_{i=1}^{n}\left|X_{i}-\mu\right|+|\mu-\widehat{m}|+\widehat{\gamma}\right]^{-(n+2)} d \mu \\
= & \int_{-\infty}^{x_{(1)}} g(\mu) d \mu+\sum_{i=1}^{n-1} \int_{x_{(i)}}^{x_{(i+1)}} g(\mu) d \mu+\int_{x_{(n)}}^{\infty} g(\mu) d \mu,
\end{aligned}
$$

where $x_{(1)}<x_{(2)}<\cdots<x_{(n)}$ are the order statistics, $\widehat{m}=x_{(n+1) / 2}$, and

$$
g(\mu)=\left[\sum_{i=1}^{n}\left|x_{(i)}-\mu\right|+\left|x_{(n+1) / 2}-\mu\right|+\widehat{\gamma}\right]^{-(n+2)} .
$$

The first integral in (C.1) is evaluated as

$$
\int_{-\infty}^{x_{(1)}} g(\mu) d \mu=\frac{1}{(n+1)^{2}}\left[n \bar{x}+\widehat{m}+\widehat{\gamma}-(n+1) x_{(1)}\right]^{-(n+1)} .
$$

Similarly, the third integral in (C.1) is

$$
\int_{x_{(n)}}^{\infty} g(\mu) d \mu=\frac{1}{(n+1)^{2}}\left[(n+1) x_{(n)}-n \bar{x}-\widehat{m}+\widehat{\gamma}\right]^{-(n+1)} .
$$

When $\mu \in\left(x_{(i)}, x_{(i+1)}\right)$, note that $g(\mu)$ is written as

$$
g(\mu)=\left[(2 i-n) \mu+n \bar{x}-2 \sum_{j=1}^{i} x_{(j)}+|\mu-\widehat{m}|+\widehat{\gamma}\right]^{-(n+2)} .
$$

Thus, if $i \leq(n-1) / 2$, it follows that

$$
\int_{x_{(i)}}^{x_{(i+1)}} g(\mu) d \mu=-\frac{1}{(2 i-n-1)(n+1)}\left(h_{1 i}\left(x_{(i+1)}\right)-h_{1 i}\left(x_{(i)}\right)\right),
$$

where

$$
h_{1 i}^{\frac{n+2}{n+1}}(\mu)=\left[(2 i-n-1) \mu+n \bar{x}+\widehat{m}-2 \sum_{j=1}^{i} x_{(j)}+\widehat{\gamma}\right]^{-(n+2)} .
$$


Similarly, if $i>(n-1) / 2$, it follows that

$$
\int_{x_{(i)}}^{x_{(i+1)}} g(\mu) d \mu=-\frac{1}{(2 i-n-1)(n+1)}\left(h_{2 i}\left(x_{(i+1)}\right)-h_{2 i}\left(x_{(i)}\right)\right)
$$

where

$$
h_{2 i}^{\frac{n+2}{n+1}}(\mu)=\left[(2 i-n+1) \mu+n \bar{x}-\widehat{m}-2 \sum_{j=1}^{i} x_{(j)}+\widehat{\gamma}\right]^{-(n+2)}
$$




\section{References}

Aerts, M., Claeskens, G., Hart, J. D., 2004. Bayesian-motivated tests of function fit and their asymptotic frequentist properties. Ann. Statist. 32 (6), $2580-2615$.

Anderson, T. W., Darling, D. A., 1954. A test of goodness of fit. J. Amer. Statist. Assoc. 49, 765-769.

Chow, Y. S., Geman, S., Wu, L. D., 1983. Consistent cross-validated density estimation. Ann. Statist. 11 (1), 25-38.

Ciardullo, R., Feldmeier, J. J., Jacoby, G. H., de Naray, R. K., Laychak, M. B., Durrell, P. R., 2002. Planetary nebulae as standard candles. xii. connecting the population i and population ii distance scales. Ap. J. 577, $31-50$

Ciardullo, R., Jacoby, G. H., Ford, H. C., Neil, J. D., 1989. Planetary nebulae as standard candles. . ii - the calibration in m31 and its companions. Ap. J. 339, 53-69.

Claeskens, G., Hjort, N. L., 2004. Goodness of fit via non-parametric likelihood ratios. Scand. J. Statist. 31 (4), 487-513.

Efromovich, S., 1999. Nonparametric curve estimation. Springer Series in Statistics. Springer-Verlag, New York, methods, theory, and applications.

Fan, J., Gijbels, I., 1996. Local polynomial modelling and its applications. Vol. 66 of Monographs on Statistics and Applied Probability. Chapman \& Hall, London.

Good, I. J., 1957. Saddle-point methods for the multinomial distribution. Ann. Math. Statist 28, 861-881.

Good, I. J., 1967. A Bayesian significance test for multinomial distributions. (With discussion). J. Roy. Statist. Soc. Ser. B 29, 399-431.

Good, I. J., 1992. The Bayes/non-Bayes compromise: a brief review. J. Amer. Statist. Assoc. 87 (419), 597-606.

Hall, P., 1987. On Kullback-Leibler loss and density estimation. Ann. Statist. 15 (4), 1491-1519.

Hart, J. D., 1997. Nonparametric smoothing and lack-of-fit tests. Springer Series in Statistics. Springer-Verlag, New York. 
Hart, J. D., 2009. Frequentist-Bayes lack-of-fit tests based on Laplace approximations. J. Stat. Theory Pract. 3 (3), 681-704.

Ingster, Y. I., Suslina, I. A., 2003. Nonparametric goodness-of-fit testing under Gaussian models. Vol. 169 of Lecture Notes in Statistics. SpringerVerlag, New York.

Jeffreys, H., 1961. Theory of probability. Third edition. Clarendon Press, Oxford.

Kass, R. E., Raftery, A., 1995. Bayes factors. J. Amer. Statist. Assoc. 90, $773-795$.

Marron, J. S., 1985. An asymptotically efficient solution to the bandwidth problem of kernel density estimation. Ann. Statist. 13 (3), 1011-1023.

Marron, J. S., Wand, M. P., 1992. Exact mean integrated squared error. Ann. Statist. 20 (2), 712-736.

Miecznikowski, J., Vexler, A., Shepherd, L., 9 2013. dbemplikegof: An r package for nonparametric likelihood ratio tests for goodness-of-fit and two-sample comparisons based on sample entropy. Journal of Statistical Software $54(3)$.

Neyman, J., 1937. Smooth test for goodness of fit. Skandinavisk Aktuarietidskrift 20, 149-199.

Rayner, J. C. W., Thas, O., Best, D. J., 2009. Smooth Tests of Goodness of Fit Using R. Wiley Series in Probability and Statistics. John Wiley \& Sons, Singapore.

Scott, D. W., 1992. Multivariate density estimation. Wiley Series in Probability and Mathematical Statistics: Applied Probability and Statistics. John Wiley \& Sons Inc., New York, theory, practice, and visualization, A Wiley-Interscience Publication.

Shapiro, S. S., Wilk, M. B., 1965. An analysis of variance test for normality: Complete samples. Biometrika 52, 591-611.

Silverman, B. W., 1986. Density estimation for statistics and data analysis. Monographs on Statistics and Applied Probability. Chapman \& Hall, London. 
Smirnov, N. V., 1944. Approximate distribution laws for random variables, constructed from empirical data. Uspekhi Matematicheskikh Nauk Issue $10,179-206$.

Thas, O., 2010. Comparing distributions. Springer Series in Statistics. Springer, New York.

Vexler, A., Gurevich, G., 2010. Empirical likelihood ratios applied to goodness-of-fit tests based on sample entropy. Comput. Statist. Data Anal. $54(2), 531-545$.

Wand, M. P., Jones, M. C., 1995. Kernel smoothing. Vol. 60 of Monographs on Statistics and Applied Probability. Chapman and Hall Ltd., London.

Zhang, X., Brooks, R. D., King, M. L., 2009. A Bayesian approach to bandwidth selection for multivariate kernel regression with an application to state-price density estimation. J. Econometrics 153 (1), 21-32.

Zhang, X., King, M. L., Hyndman, R. J., 2006. A Bayesian approach to bandwidth selection for multivariate kernel density estimation. Comput. Statist. Data Anal. 50 (11), 3009-3031. 